\title{
Etiologies and Adverse Outcomes of Fetuses with Short Femur Length Based on Proportion and Percentile Categorization
}

\author{
Jianan Li, BS ${ }^{a, 1}$, Hong Xu, MD ${ }^{a, 1}$, Mei Shen, BS ${ }^{a}$, Shiyu Li, BS ${ }^{a}$, Longxia Wang, MD ${ }^{a, *}$, Yanping Lu, MD ${ }^{b, *}$, \\ Qiuyang $\mathrm{Li}, \mathrm{MD}^{a \text {,* }}$ \\ ${ }^{a}$ Department of Ultrasound, First Medical Center, Chinese PLA General Hospital, Beijing, China; ${ }^{b}$ Department of Gynecology and \\ Obstetrics, First Medical Center, Chinese PLA General Hospital, China
}

Received June 2, 2021; revision received June 28, 2021; accepted July 5, 2021

Objective: To compare the etiologies and adverse outcomes of pregnancies with short fetal femur length (FL) categorized based on relative proportion and percentile placement.

\begin{abstract}
Methods: Fetuses $(n=254)$ with short FL measured by ultrasound were classified into four groups: severe and disproportionate short FL (group A); severe and proportionate short FL (group B); mild and disproportionate short FL (group C); mild and proportionate short FL (group D). Etiologies and outcomes of pregnancies were compared between groups.

Results: A larger percentage of skeletal dysplasia occurred in group A (28.57\%) than in the other three groups (group B: 1.85\%; group C: 4.08\%; group D: 0). Groups A and B also had higher rates (group A: 40.82\%, group B:33.33\%) of fetal growth restriction (FGR) than groups $\mathrm{C}$ and $\mathrm{D}$ (group C: $4.08 \%$; group D:3.92\%). No difference in chromosomal and genetic anomalies was detected among groups $(P=0.307)$; however, the rate of chromosomal and genetic testing in group A was higher than in group D $(P=0.001)$. Group A $(57.14 \%)$ had a significantly lower rate of live birth than the other three groups, and group D (98.04\%) had a higher live birth rate than groups B (87.04\%) and C (83.67\%). Considering only living fetuses, higher rates of preterm birth $(P<0.001)$, low birth weight (LBW) $(P<0.001)$, neonatal intensive care unit (NICU) admission or pediatric hospital referral $(P=0.004)$, Apgar score $(1 \mathrm{~min} / 5 \mathrm{~min}) \leqslant 7(P<0.001)$ and fetal distress or neonatal asphyxia $(P=0.004)$ were found in group A compared to group D.
\end{abstract}

Conclusion: Fetuses with severe and disproportionate short FL should be flagged for possible skeletal dysplasia. Fetuses with severe short FL should be watched for FGR prenatally. Invasive prenatal tests are recommended for fetuses with severe and disproportionate FL but not recommended for those with mild and proportionate short FL. Outcomes of fetuses with severe and disproportionate short FL were worse than that of fetuses with mild and proportionate short FL.

Key words: Short fetal femur length; Disproportionate; Etiologies; Outcomes

Advanced Ultrasound in Diagnosis and Therapy 2022; 01: 007-013

DOI: 10.37015/AUDT.2021.210015

$\mathrm{T}$ The only long bone measurement required for the assessment of fetal growth is femur length (FL) according to the most recent guidelines of the International Society of Ultrasound in Obstetrics and
Gynecology (ISUOG) [1]. Short FL may be associated with fetal growth restriction (FGR), skeletal dysplasia, and chromosomal and gene anomalies. Ample evidence indicates an association between short FL and adverse

\footnotetext{
${ }^{1}$ Jianan Li and Hong Xu as co-first authors contributed equally to the study.

" Corresponding author: Department of Ultrasound, First Medical Center, Chinese PLA General Hospital, 28 Fuxing Road, Beijing 100853, China

e-mails: LXWang,13693685342@163.com; YP Lu,luyp301@163.com; QY li,Liqiuyang0925@163.com unrestricted use, distribution and reproduction in any medium provided that the original work is properly attributed.
} 
outcomes [2]. Nonetheless, short FL may also reflect inaccurate dating or a normal variant in constitutionally small fetuses [2]. As a result, the diagnosis of short FL makes prenatal counselling difficult.

We introduce here new parameters to distinguish short FL in more detail, which may help to class fetuses with short FL into those where adverse outcomes may be more likely and those with a more positive prognosis. Short FL is variably defined as less than the 3rd to less than the $10^{\text {th }}$ percentile for a given gestational age $[2,3]$. Etiologies and outcomes of fetuses with short FL diagnosed at different percentiles have not been evaluated in controlled studies. The only study we were able to find regarding the degree of short femur was a comparison of chromosomal and genetic analysis between fetuses with a FL 2-4 standard deviations (SDs) below the GA (gestational age) mean and a FL > 4 SDs below the mean [4]; other etiologies and outcomes were not involved in previous study.

An individual with an altered upper/lower segment $(\mathrm{U} / \mathrm{L})$ ratio is described as having a disproportionate short stature. Most individuals with disproportionate short stature have skeletal dysplasia, while those with proportionate short stature are more likely to have endocrine, nutritional, or genetic disorders [5]. Other ratios regarding FL have been used in previous studies. Fetal femur length can be considered shortened when the biparietal diameter to femur length $(\mathrm{BPD} / \mathrm{FL})$ ratio is above $1.5[5,6]$. A FL to abdominal circumference $(\mathrm{FL} / \mathrm{AC})$ ratio $<0.16$ has been described as one feature of achondroplasia [7], but no controlled study has been performed regarding proportionate or disproportionate short FL. Therefore, we measured severity of short FL using percentile and proportion as classifying criteria and evaluated the etiology and outcome of fetuses from different categories.

\section{Materials and Methods}

\section{Patients}

The study was approved by Ethics Committee of the People Liberation Army (PLA) General Hospital. This was a retrospective cohort analysis conducted at First Medical Center of Chinese PLA General Hospital in Beijing. All singleton pregnancies with a diagnosis of fetal femur below the $10^{\text {th }}$ percentile at $14-42$ weeks gestation from April 2017 to February 2021 were enrolled. No twin pregnancies were included in our study. Those patients without sufficient data for our study were excluded. Finally, a total of 254 cases were included in our study.

\section{Four study groups were classified as follows}

Group A: fetuses with FL below percentile 3 and a lower $\mathrm{FL} / \mathrm{BPD}$ ratio than expected ( $\mathrm{FL}<3 \%$ and lower FL/BPD ratio); defined as severe and disproportionate short FL.

Group B: fetuses with FL below percentile 3 and a normal $\mathrm{FL} / \mathrm{BPD}$ ratio $(\mathrm{FL}<3 \%$ and normal $\mathrm{FL} / \mathrm{BPD}$ ratio); defined as severe and proportionate short FL.

Group C: fetuses with FL below percentile 10 (but $\geqslant$ percentile 3) and lower FL/BPD ratio than expected (FL at $3-10 \%$ and lower FL/BPD ratio); defined as mild and disproportionate short FL.

Group D: fetuses with FL below percentile 10 (but $\geqslant$ percentile 3 ) and normal $\mathrm{FL} / \mathrm{BPD}$ ratio (FL at $3-10 \%$ and normal FL/ BPD ratio); defined as mild and proportionate short FL.

Of the 254 patients, groups A, B, C and D included 49 (19.29\%), 54 (21.26\%), 49 (19.29\%) and 102 (40.16\%) cases, respectively.

\section{Data and definitions}

All ultrasound scans for FL measurements were performed using a transducer of SC6-1U with Mindray machine and a transducer of CA3-10A with SAMSUNG machine by two experts in obstetrics and gynecology ultrasound to minimize the possibility of measurement error. Where multiple ultrasound exams were available, the ultrasound report at the latest gestational age in which $\mathrm{FL}<10^{\text {th }}$ percentile was diagnosed was adopted for use. All ultrasound reports, maternal and neonatal medical records in the electronic medical database of the hospital were searched and information needed for the study was completed by telephone follow-up.

The data including maternal demographics, fetal biometric parameters, malformation, soft markers and chromosomal abnormalities, obstetrical and perinatal outcomes was collected for analysis.

\section{Etiology}

(1) Skeletal dysplasia; Other malformation: Main malformations except skeletal dysplasia; Soft markers: softer markers without skeletal dysplasia or other malformations. (2) Fetal growth restriction (FGR) defined according to the Delphi consensus criteria as recommended by the ISUOG guidelines [8]. (3) Chromosomal and genetic abnormalities. Amniotic fluid obtained by amniocentesis or cord blood obtained by cord puncture was used to perform karyotype analysis, chromosomal microarray testing (CMA), genetic mutation testing or whole exome sequencing.

\section{Outcomes}

(1) Live birth. (2) Still birth included miscarriage, 
intrauterine demise, and termination of pregnancy (TOP). Miscarriage was defined as a clinically recognized pregnancy loss before 28 weeks of gestation; intrauterine demise was defined as fetal death after 28 weeks' gestation and prior to delivery. (3) Cesarean delivery: scheduled or emergency cesarean delivery. (4) Preterm birth: delivery before 37 weeks gestation. (5) Low birth weight (LBW): neonates with a birth weight $<2500$ g. (6) NICU admission: neonatal intensive care unit (NICU) admission or pediatric hospital referral. (7) Apgar score $\leqslant 7$ : recorded as positive if either $1 \mathrm{~min}$ or $5 \mathrm{~min}$ Apgar score result $\leqslant 7$ was recorded. (8) Fetal distress or neonatal asphyxia.

\section{Statistical analysis}

Pearson $\chi^{2}$ was used to analyze differences among the four groups. Fisher exact test was used to evaluate the difference among four groups in special types of skeletal dysplasia. Differences were considered statistically significant if $P$ value $<0.05$. Bonferroni was used to perform pairwise comparison between groups; a $P$ value $<0.0083$ was considered statistically significant for these comparisons. SPSS 23 (IBM, Chicago, USA) was used for stastical analysis.

\section{Results}

There was no significant difference among the groups in the demographic characteristics of the pregnancies as presented in Table 1.
Etiologies in our study involved skeletal dysplasia, FGR, and chromosomal and genetic anomalies. We identified a larger percentage of skeletal dysplasia in group A (28.57\%), compared to groups B (1.85\%), C $(4.08 \%)$ or D (0) (Table 2). No difference in skeletal dysplasia among groups B, C and D was detected. Table 3 indicates specific types of skeletal dysplasia observed. Significant difference existed in achondroplasia $(P=0.003)$ and femoral curvature $(P=0.014)$ among the four groups, but no difference was demonstrated using pairwise comparisons. Differences in other malformations among the four groups were detected $(P=0.025)$ but no differences could be verified using pairwise comparisons. The presence of soft markers did not differ significantly among the groups $(P=0.773)$. Group A $(P=40.82 \%)$ had a significantly higher FGR rate than groups $\mathrm{C}(4.08 \%)$ and $\mathrm{D}(3.92 \%)$, while group B (33.33\%) also had a higher risk of FGR than groups C and $\mathrm{D}$. Moreover, no statistically significant difference was detected between groups $\mathrm{A}$ and $\mathrm{B}$, nor between groups $\mathrm{C}$ and $\mathrm{D}$. As presented in Table 4, no significant difference in chromosomal and genetic anomalies was detected among groups $(P=0.307)$. The chromosomal and genetic aberrations observed in our study are shown in Table 5. The rates of chromosomal or genetic testing among groups differed significantly $(P=0.012)$, with group A significantly higher than group D $(38.78 \%$ vs. $14.71 \%, P=0.001)$.

Table 1 Demographic characteristics of study population

\begin{tabular}{lccccc}
\hline Item & Group A $(n=49)$ & Group B $(n=54)$ & Group C $(n=49)$ & Group D $(n=102)$ & $P$ value \\
\hline Maternal age $\geqslant 35$ ys & 7 & 18 & 12 & 33 & 0.085 \\
Maternal tobacco uses or history of bleeding & 31 & 30 & 25 & 60 & 0.420 \\
\hline Pregnancy hypertension disease & 2 & 4 & 4 & 7 & 0.861 \\
Preeclampsia & 4 & 5 & 4 & 3 & 0.188 \\
\hline Pregnancy with hypothyroidism & 2 & 9 & 8 & 17 & 0.139 \\
Gestational diabetes & 5 & 11 & 7 & 18 & 0.570 \\
\hline History of adverse pregnancy & 7 & 6 & 4 & 13 & 0.248 \\
\hline Myoma of uterus & 2 & 3 & 9 & 23 & 0.814 \\
\hline Scared uterine & 9 & 9 & & & \\
\hline
\end{tabular}

Table 2 Ultrasound findings of the fetuses in four groups

\begin{tabular}{|c|c|c|c|c|c|}
\hline Item & Group A $(n=49)$ & Group B $(n=54)$ & Group C $(n=49)$ & Group D $(n=102)$ & $P$ value \\
\hline Skeletal dysplasia & 14 & $1^{*}$ & $2^{\ddagger}$ & $0^{*}$ & $<0.001$ \\
\hline Other malformations & 12 & 6 & 13 & 11 & 0.025 \\
\hline Soft markers & 4 & 2 & 4 & 7 & 0.773 \\
\hline Fetal growth restriction & 20 & 18 & $2^{\ddagger}+\dagger$ & $4^{t, \dagger}$ & $<0.001$ \\
\hline
\end{tabular}

${ }^{\ddagger}$ Compared with group A, $P<0.001 ;{ }^{\dagger}$ Compared with group B, $P<0.001$. 
Table 3 Skeletal dysplasia in four groups

\begin{tabular}{lcccc}
\hline Item & Group A $(n=49)$ & Group B $(n=54)$ & Group C $(n=49)$ & Group D $(n=102)$ \\
\hline Achondroplasia & 4 & 0 & 0 & 0 \\
Osteogenesis imperfect & 1 & 0 & 0.003 & 0 \\
Thanatophoric & 2 & 0 & 0 & 0 \\
Talipes and valgus & 2 & 1 & 2 & 0 \\
Short limbs & 2 & 0 & 0 & 0 \\
Femoral curvature & 3 & 0 & 0 & 0.073 \\
\hline
\end{tabular}

Table 4 Chromosomal and genetic results of the fetuses in four groups

\begin{tabular}{|c|c|c|c|c|c|c|c|c|c|}
\hline \multirow{2}{*}{ Item } & \multicolumn{2}{|c|}{ Group A } & \multicolumn{2}{|c|}{ Group B } & \multicolumn{2}{|c|}{ Group C } & \multicolumn{2}{|c|}{ Group D } & \multirow{2}{*}{ - $P$ value } \\
\hline & Total number & Abnormal & Total number & Abnormal & Total number & Abnormal & Total number & Abnormal & \\
\hline Karyotype analysis & 5 & 2 & 7 & 0 & 8 & 2 & 11 & 1 & 0.229 \\
\hline CMA testing & 12 & 0 & 10 & 3 & 3 & 1 & 7 & 1 & 0.211 \\
\hline Genetic testing & 10 & 3 & 3 & 0 & 3 & 2 & 1 & 1 & 0.180 \\
\hline All chromosomal and genetic results & 19 & 5 & 14 & 3 & 11 & 5 & 15 & 2 & 0.307 \\
\hline
\end{tabular}

CMA, chromosomal microarray analysis.

Table 5 chromosomal and genetic anomalies in all groups

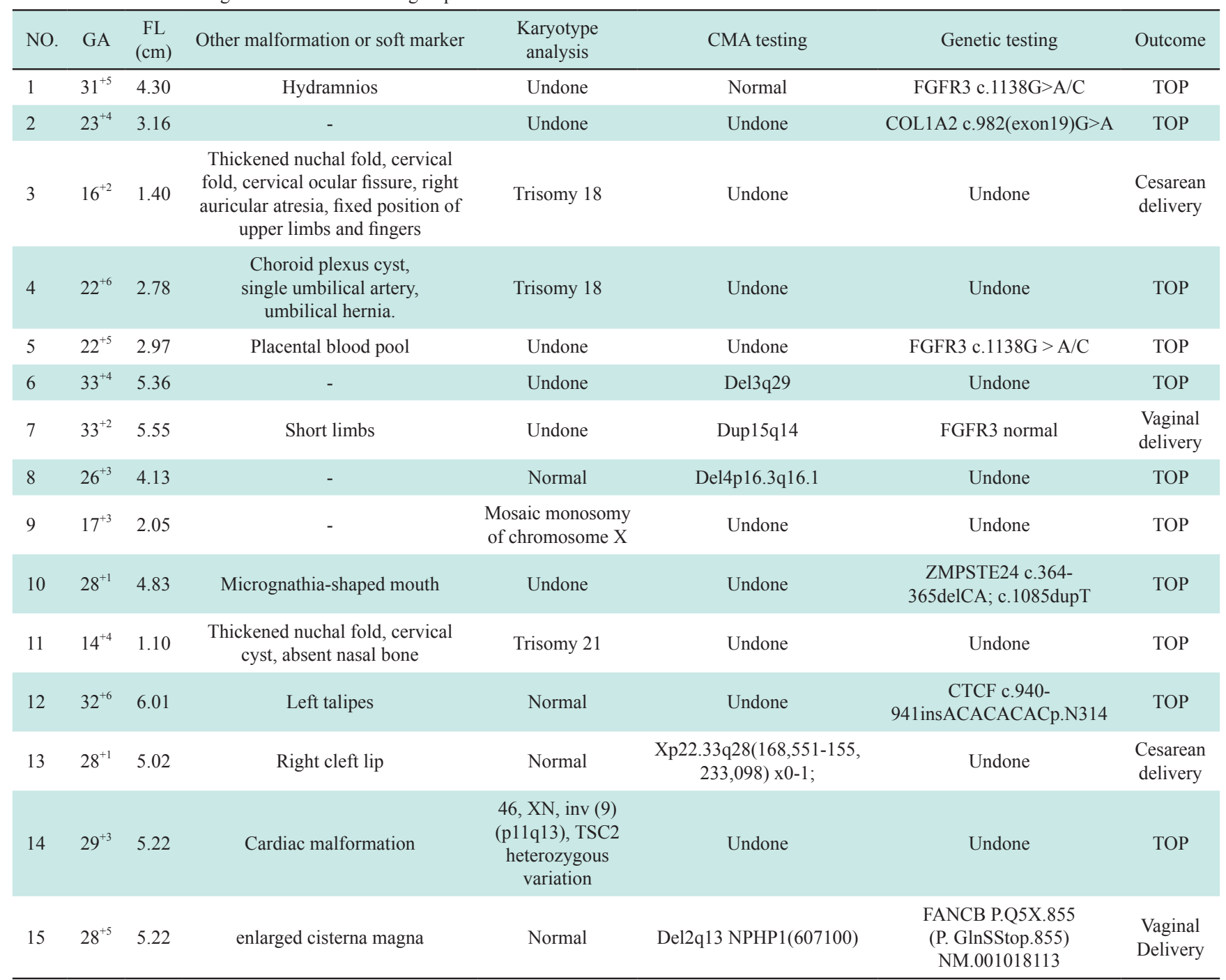

GA, gestational age; FL, femur length; TOP, terminal of pregnancy; NO.1-5 were cases in group A; NO.6-8 were cases in group B; NO.9-13 were cases in group. C; NO.14-15 were cases in group D. 
As shown in Table 6, significant difference in live birth rates existed among the groups. Group A (57.14\%) showed a significantly lower rate of live births compared to the other three groups. In addition, group D (98.04\%) had a significantly higher rate of live birth than groups B (87.04\%) and C (83.67\%). When considering only living fetuses, significantly higher detection rates of preterm birth, NICU admission or pediatric hospital referral, Apgar score $\leqslant 7$, and fetal distress or neonatal asphyxia were found in group A than in group D (Table 7). Moreover, group A (57.14\%) showed a higher risk of LWB than groups C (4.88\%) and D (4.00\%), while group D showed a lower risk of LWB than group B (17.02\%).

Table 6 Outcomes of all fetuses in four groups

\begin{tabular}{lccccc}
\hline Item & Group A $(n=49)$ & Group B $(n=54)$ & Group C $(n=49)$ & Group D $(n=102)$ & $P$ value \\
\hline Live birth & 28 & $47^{\ddagger}$ & $41^{\ddagger}$ & $100^{\ddagger \dagger}$ & $<0.001$ \\
Still birth & 21 & 7 & 8 & 2 & $<0.001$ \\
\hline
\end{tabular}

${ }^{\ddagger}$ Compared with group A, $P<0.001 ;{ }^{\dagger}$ Compared with group C, $P<0.001$.

Table 7 Adverse outcomes of live births of four groups

\begin{tabular}{|c|c|c|c|c|c|}
\hline Item & Group A $(n=28)$ & Group B $(n=47)$ & Group C $(n=41)$ & Group $\mathrm{D}(n=100)$ & $P$ value \\
\hline Cesarean delivery & 18 & 27 & 21 & 42 & 0.116 \\
\hline Preterm birth & 9 & 4 & 5 & $6^{\ddagger}$ & $<0.001$ \\
\hline LBW & 10 & 8 & $2 \ddagger$ & $4^{t+\dagger}$ & $<0.001$ \\
\hline NICU admission & 12 & 14 & 11 & $17^{\ddagger}$ & 0.032 \\
\hline Apgar score $(1 \mathrm{~min} / 5 \mathrm{~min}) \leqslant 7$ & 4 & 1 & 1 & $0^{*}$ & $<0.001$ \\
\hline Fetal distress or neonatal asphyxia & 15 & 16 & 12 & $26^{\ddagger}$ & 0.049 \\
\hline
\end{tabular}

LBW, low birth weight; NICU admission, neonatal intensive care unit admission or pediatric hospital refer; ${ }^{\ddagger}$ Compared with group A, $P<0.0083$; ${ }^{\dagger}$ Compared with group B, $P<0.0083$.

\section{Discussion}

\section{Etiology}

Skeletal dysplasia associated with short FL mainly included achondroplasia (ACH), osteogenesis imperfecta (OI), thanatophoric (TD), and talipes [6,9]. Achondroplasia is the most common nonlethal skeletal dysplasia and is related to a mutation in the FGFR3 gene [9]. Four patients with achondroplasia were diagnosed in our study. Two of them presented the mutation of c.1138G $>$ A affecting FGFR3. Osteogenesis imperfecta (OI) is a heritable disorder of bone delineated into four types [6]. The mutation of COL1A2 is associated with OI [4]. One fetus with OI was diagnosed in our study, with genetic results presented as c.982 (exon19) G>A on COL1A2. Thanatophoric Dysplasia (TD), the most common lethal skeletal dysplasia, is always caused by autosomal dominant mutations in the FGFR3 gene. Fetuses with TD always die in utero or a few days after birth and respiratory distress is the main cause [10]. TD was observed in two fetuses in our study; neither had any invasive chromosomal or genetic tests. Talipes equinovarus (clubfoot) is one of the most common congenital anomalies, with the affected foot in adduction, supination, varus or valgus position. The deformity may be isolated, associated with additional structural malformations, or associated with chromosomal or genetic anomalies [10]. Four fetuses with talipes equinus and one fetus with talipes valgus were present in our study. The fetus with talipes valgus also had a genetic anomaly, a mutation on the CTCF gene, and normal CMA result. Two patients with short limbs and three patients with femoral curvature were also observed in our study. Our study demonstrates that skeletal dysplasia is more likely to be present in fetuses with severe and disproportionate short FL. Fetuses with severe and disproportionate short FL should thus be considered at risk of skeletal dysplasia during ultrasound scanning. The use of prenatal 3D-ultrasound can be helpful for detecting special features of skeletal dysplasia [11]. Skeletal dysplasia is always related to a gene mutation, and the rate of chromosomal or genetic tests is higher in fetuses with severe and disproportionate short FL. Therefore, we recommend invasive genetic tests in all fetuses with severe and disproportionate short FL. In contrast, given the lower rate of skeletal dysplasia 
and adverse outcomes, we do not recommend invasive prenatal tests in pregnancies where the fetus has mild and proportionate short FL.

The association between short FL and subsequent outcomes of placenta dysfunction such as FGR, IUGR (intrauterine growth retardation) and SGA (small for gestational age), has been well-demonstrated in the literature. Pregnancies with a short fetal femur length of less than the $10^{\text {th }}$ percentile ( $\mathrm{FL}<10^{\text {th }}$ percentile) have higher incidence of FGR compared to pregnancies with a normal femur length [12]. The prevalence of IUGR or SGA is higher in fetuses with short femur length ( $\mathrm{FL}<5^{\text {th }}$ percentile) compared to normal fetuses $[2,7,12,13]$. We use the definition of FGR as the newest ISUOG guideline recommended and showed a relation between short FL and FGR. In addition, our findings showed that fetuses with severe short FL, proportionate or disproportionate, were more likely to experience FGR compared to those with mild short FL, whether proportionate or not. In fetuses with severe short FL, enhanced monitoring of fetal growth and attention to adverse outcomes of placental dysfunction, such as chronic fetal hypoxia or early preeclampsia is necessary.

The effect of chromosomal analysis and gene sequencing has been demonstrated in previous studies [4]. Short FL is a sonographic soft marker of aneuploidy $[4,14]$. In our study, trisomy 21 , trisomy 18 and mosaic monosomy of chromosome X were detected. Our study supports the contribution of short FL to chromosomal aberration detected by CMA. CMA aberration on chromosomes 2, 3, 4, 15 and $\mathrm{X}$ were revealed in our study. We identified a pathogenic $868.1 \mathrm{~Kb}$ deletion on $2 q 13$ that involved NPHP1 gene (607100). Mutation in NPHP1 has been reported in patients with renal failure, intellectual disability, growth retardation, autism, and language delay [4]. We also detected a variation on $\mathrm{Xp} 22.33$ which is associated with the short stature homeobox gene (SHOX.) [4]. Genetic mutations of FGFR3 and COL1A2 were observed in our study. In addition, mutations on ZMPSTE (associated with fatal lethal restrictive dermatosis), CTCF and FANCB genes were observed. The association between short FL and these mutations has not been verified, but the knowledge will be valuable for further studies.

Except for proportion and percentile, early versus late sonographic diagnosis of short femur length was also related to different etiologies and outcomes. The incidence of abnormal karyotype and skeletal dysplasia is higher when short FL is diagnosed earlier in gestation, while the incidence of being SGA, IUGR and the rate of live births are significantly increased when short $\mathrm{FL}$ is diagnosed later during pregnancy [5].

\section{Adverse outcomes}

A connection between short FL at different percentiles and adverse outcomes has been noted in previous literature. The risk of miscarriage, TOP and still birth increased significantly in fetuses with a $\mathrm{FL}<$ percentile 5 $[10,13,14]$. Fetuses with a short FL of $<$ percentile 10 are also more likely to have intrauterine death [13]. Worsening of femoral length was the main reason for TOP in fetuses with short FL [15]. Our study demonstrates outcome differences based on percentile classifications of short FL. Fetuses with severe and disproportionate short FL had a significantly lower rate of live birth than fetuses with mild and proportionate short FL.

Different standards of short FL were also used when considering the adverse outcomes of only living fetuses. Pregnancies with a short $\mathrm{FL}<10^{\text {th }}$ percentile are more likely to have a preterm delivery between 34 and 37 weeks [13]. Pregnancies with a short FL $<5^{\text {th }}$ percentile are also more likely to have preterm delivery $[7,13]$. Low birth weight, Apgar $\leqslant 7$ at $5 \mathrm{~min}$ and NICU admission were all significantly associated with short $\mathrm{FL}<5^{\text {th }}$ percentile $[2,7]$. Significantly higher detection rates of preterm birth, LBW, NICU admission or pediatric hospital referral, Apgar score $(1 \mathrm{~min} / 5 \mathrm{~min}) \leqslant 7$ and fetal distress or neonatal asphyxia occurred in fetuses with severe and disproportionate short FL, compared to those with mild and proportionate short FL.

\section{Limitations}

FL is not a measurement required in the first trimester. But some fetuses with short FL accompanied with other structural or chromosomal anomalies were observed in the first trimester. These individuals were not included in our study, as they lacked other necessary ultrasound parameters. We thus only discussed the value of short FL diagnosed in the second and third trimester. Fetuses with normal FL were not included in our study as a control group. Race also affects the length of the femur and the humerus [16]. South Asian fetuses have smaller abdominal circumference (AC), but larger FL than Europeans and Ethnic East Asians had lower birth weight [17]. The study population of our study includes only Asians, and difference in etiologies and outcomes between fetuses with short FL of different races cannot be evaluated.

\section{Conclusion}

Fetuses with severe and disproportionate short FL should be considered at risk of skeletal dysplasia. All fetuses with severe short FL should be monitored for FGR during the prenatal terms. Invasive prenatal tests are recommended for fetuses with severe and disproportionate FL but not recommended for those with mild and proportionate short FL. Fetuses with severe 
and disproportionate short FL had significantly worse outcomes than those with mild and proportionate short fetuses.

\section{Acknowledgment}

The research was supported by The National Key Research and Development Program of China, No.2018YFC1002202 and Capital characteristic Program No.Z171100001017141.

\section{Conflict of Interests}

The authors have no conflicts of interest in relation to this work.

\section{References}

[1] Salomon LJ, Alfirevic Z, Berghella V, Bilardo C, HernandezAndrade E, Johnsen SL, et al. Practice guidelines for performance of the routine mid-trimester fetal ultrasound scan. Ultrasound Obstet Gynecol 2011; 37: 116-126.

[2] Aviram A, Bardin R, Wiznitzer A, Yogev Y, Hadar E. Midtrimester isolated short femur length as a predictor of adverse pregnancy outcome. Fetal Diagn Ther 2015; 38: 205-211.

[3] Kaijomaa M, Ulander VM, Ryynanen M, Stefanovic V. Risk of adverse outcomes in euploid pregnancies with isolated short fetal femur and humerus on second-trimester sonography. $J$ Ultrasound Med 2016; 35: 2675-2680.

[4] Liu J, Huang L, He Z, Lin S, Wang Y, Luo Y. Clinical value of genetic analysis in prenatal diagnosis of short femur. Mol Genet Genomic Med 2019; 7: e978.

[5] D'Ambrosio V, Vena F, Boccherini C, Di Mascio D, Squarcella A, Corno S, et al. Obstetrical and perinatal outcomes in fetuses with early versus late sonographic diagnosis of short femur length: A single-center, prospective, cohort study. Eur J Obstet Gynecol Reprod Biol 2020; 254: 170-174.

[6] Krakow D, Rimoin DL. The skeletal dysplasias. Genet Med 2010; 12: $327-341$.

[7] D'Ambrosio V, Vena F, Marchetti C, Di Mascio D, Perrone S,
Boccherini C, et al. Midtrimester isolated short femur and perinatal outcomes: A systematic review and meta-analysis. Acta Obstet Gynecol Scand 2019; 98: 11-17.

[8] Abuhamad A, Martins JG, Biggio JR. Diagnosis and management of fetal growth restriction: the SMFM guideline and comparison with the ISUOG guideline. Ultrasound Obstet Gynecol 2021; 57: 880-883.

[9] Khalil A, Morales-Roselló J, Morlando M, Bhide A, Papageorghiou A, Thilaganathan B. Widening of the femoral proximal diaphysis-metaphysis angle in fetuses with achondroplasia. Ultrasound Obstet Gynecol 2014; 44: 69-75.

[10] Di Mascio D, Buca D, Khalil A, Rizzo G, Makatsariya A, Sileo F, et al. Outcome of isolated fetal talipes: A systematic review and metaanalysis. Acta Obstet Gynecol Scand 2019; 98: 1367-1377.

[11] Jung M, Park SH. Genetically confirmed thanatophoric dysplasia with fibroblast growth factor receptor 3 mutation. Exp Mol Pathol 2017; 102: 290-295.

[12] Ventura W, Prieto-Sánchez MT, Delgado JL, Pertegal M, López A, Checa R, et al. Short femur in the second trimester scan is related to maternal preeclampsia and small for gestational age newborns. Fetal Diagn Ther 2020; 47: 615-623.

[13] Goetzinger KR, Cahill AG, Macones GA, Odibo AO. Isolated short femur length on second-trimester sonography: a marker for fetal growth restriction and other adverse perinatal outcomes. $J$ Ultrasound Med 2012; 31: 1935-1941.

[14] Mathiesen JM, Aksglaede L, Skibsted L, Petersen OB, Tabor A. Danish fetal medicine study group. Outcome of fetuses with short femur length detected at second-trimester anomaly scan: a national survey. Ultrasound Obstet Gynecol 2014; 44: 160-165.

[15] Hu T , Tian T , Zhang Z , Wang J , Liu S. Prenatal chromosomal microarray analysis in 2466 fetuses with ultrasonographic soft markers: a prospective cohort study. American Journal of Obstetrics and Gynecology 2020.

[16] Harper LM, Gray D, Dicke J, Stamilio DM, Macones GA, Odibo AO. Do race-specific definitions of short long bones improve the detection of down syndrome on second-trimester genetic sonograms? J Ultrasound Med 2010; 29: 231-235.

[17] Sletner L, Rasmussen S, Jenum AK, Nakstad B, Jensen OH, Vangen S. Ethnic differences in fetal size and growth in a multi-ethnic population. Early Hum Dev 2015; 91: 547-554. 